\title{
Shock: A possible presenting manifestation of autoimmune polyendocrine syndrome type II
}

\author{
Subodh Banzal, Abhishek Singhai
}

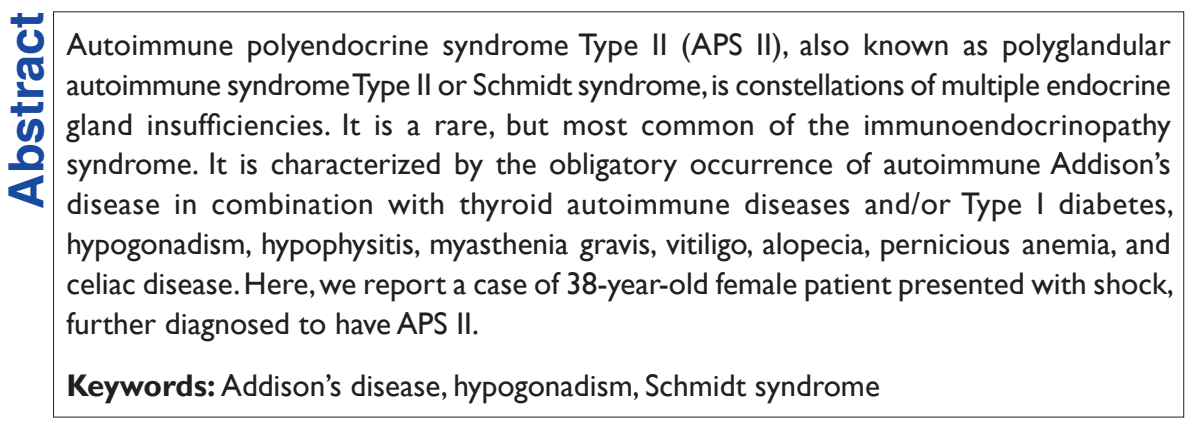

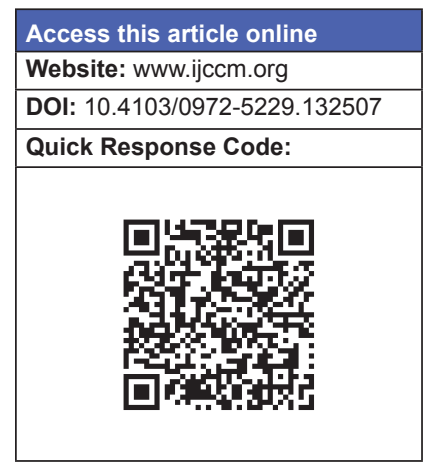

\section{Introduction}

Historically, the interest in these syndromes began in the $19^{\text {th }}$ century and essentially focused on the adrenal cortex. In 1849, Thomas Addison first described the clinical and pathologic features of adrenocortical failure in patients who also appeared to have coexisting pernicious anemia. In 1981, Neufeld and colleagues distinguished two major polyglandular autoimmune (PGA) syndromes, and other authors subsequently began to add to our knowledge of these conditions. In 2004, Eisenbarth and Gottlieb extended the discussion on the classification of these syndromes. The frequency of Type II PGA in humans is rare, being described in about 1.4-4.5/100,000 inhabitants.

\section{Case Report}

The case we present here is a 38-year-old female patient who presented to emergency department in a state of shock with a history of recurrent vomiting, abdominal discomfort, and mild fever since last 3 days. On direct

\section{From:}

Department of Medicine, Sri Aurobindo Medical College, Indore, Madhya Pradesh, India

\section{Correspondence:}

Dr. Abhishek Singhai, Department of Medicine, Sri Aurobindo Medical College, Sanwer Road, Indore, Madhya Pradesh, India.

E-mail: drabhisheksinghai@gmail.com inquiry, she gave a history of anorexia, weight loss, postural blackouts, and generalized weakness since last 6 months. She also noticed darkening of skin color since last 1 year. She was mother of two children and had amenorrhea since last 8 years. On examination patient was thin built with body mass index 22. She had dark skin with mucosal pigmentation. Her pulse was feeble and blood pressure $60 \mathrm{mmHg}$ systolic. Her breast size was B4 and pubic hairs were scanty. Other system examinations were normal. There was no history of similar illness in her family members.

Investigations are shown in Table 1. Based on these investigations, we found that patient had multiple endocrine insufficiencies, including Addison's disease (decreased cortisol level and increased adrenocorticotropic hormone level), hypothyroidism (decreased free $\mathrm{T}_{4}$ and increased thyroid-stimulating hormone) and primary ovarian failure (amenorrhea with increased follicle-stimulating hormone and luteinizing hormone). Patient was initially treated with intravenous fluids, antibiotics, and dopamine infusion, but the patient's condition did not improve. After investigations showed Addison's disease and hypothyroidism intravenous hydrocortisone and oral L-thyroxine were started. She improved with these measures and could be discharged after 1 week on oral prednisolone, fludrocortisone, L-thyroxine, estrogen 


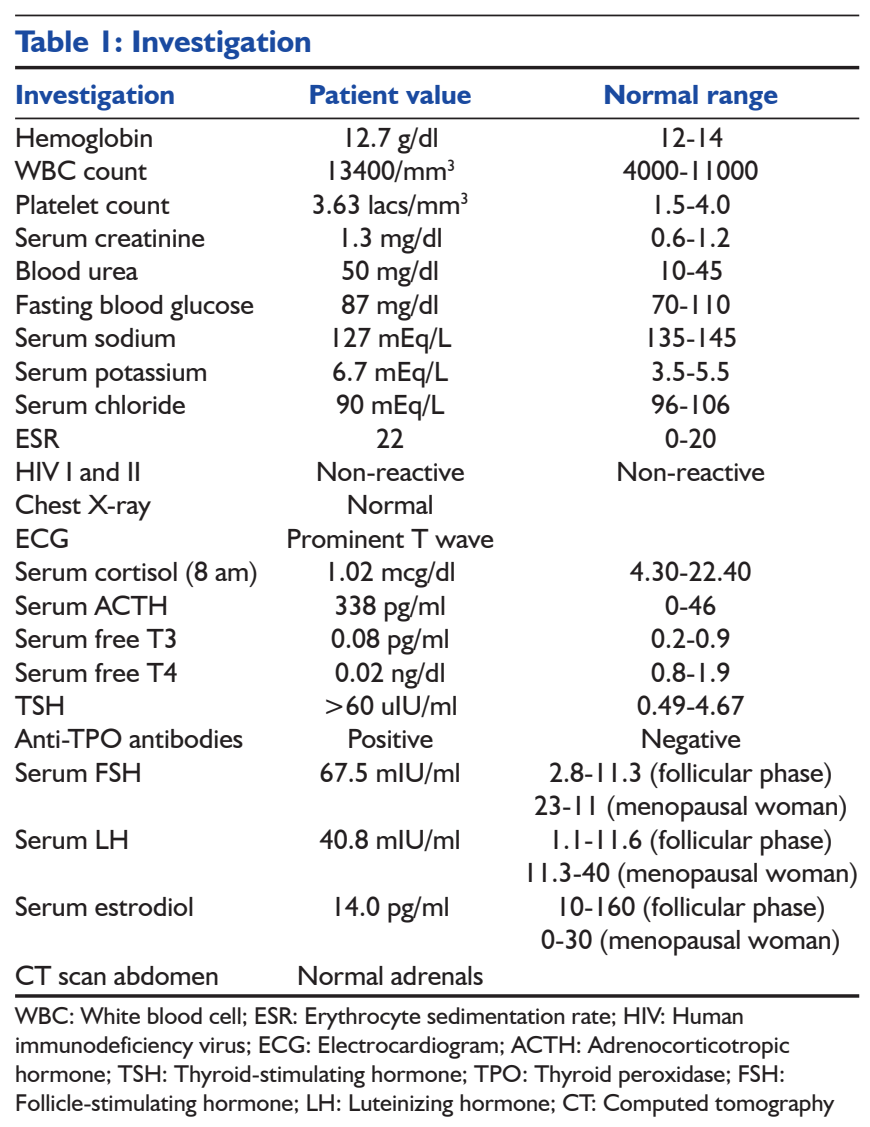

and progesterone. She also developed regular menstrual cycle on estrogen-progesterone cyclical therapy. Her clinical condition remained normal on above therapy. Pigmentation of skin and mucous membrane also decreased.

\section{Discussion}

Autoimmune polyendocrine syndrome Type II (APS II) occurs primarily in adulthood, usually around the $3^{\text {rd }}$ and $4^{\text {th }}$ decades of life. Middle-aged women have shown an increased prevalence of APS II. It is associated with human leukocyte antigen (HLA)-DR3 and/ or HLA-DR4 haplotypes, and the pattern of inheritance is autosomal dominant with variable expressivity. ${ }^{[1]}$

The pathogenesis of APS II is poorly understood. Some degree of genetic susceptibility must exist in the individual. ${ }^{[2]}$ The individual is then exposed to the autoimmune trigger, which could be an environmental or intrinsic factor. The trigger mimics the molecular structure of a self-antigen. Next, a subclinical phase of active production of organ-specific auto antibodies occurs. APS II consists of Addison's disease plus either an autoimmune thyroid disease or Type I diabetes mellitus associated with hypogonadism, pernicious anemia, celiac disease, recent primary biliary cirrhosis, myasthenia gravis, Parkinson disease, vitiligo, alopecia and seronegative arthritis. The clinical features consist of a constellation of the individual endocrinopathies. ${ }^{[3]}$ Clinical history and examination suggesting evidence of $>1$ endocrine deficiency should prompt testing, to include serum autoantibody screening and an evaluation of end-organ function. ${ }^{[4]}$

The screening panel includes autoantibodies to 21-hydroxylase, 17-hydroxylase, thyroid peroxidase, glutamic acid decarboxylase, islet cells, antitissue transglutaminase antibodies, parietal cell and anti-intrinsic factor antibodies..$^{[5]}$

Currently, the treatment of the APSs is dictated by the individual disorders. With the exception of celiac disease and Graves disease, the mainstay of treatment is primarily hormonal replacement therapy. ${ }^{[6]}$

\section{Conclusion}

Clinically overt syndrome is considered only the tip of the iceberg, since latent forms are much more frequent. Organ-specific autoantibody screening in patients with monoglandular autoimmune endocrinopathies facilitates the identification of those at risk of developing a future APS. Early identification and treatment of associated autoimmune endocrine disease may be critical and even life-saving as shown in our patient.

\section{References}

1. Obermayer-Straub P, Manns MP. Autoimmune polyglandular syndromes. Baillieres Clin Gastroenterol 1998;12:293-315.

2. Ramos-Lopez E, Lange B, Kahles H, Willenberg HS, Meyer G, Penna-Martinez M, et al. Insulin gene polymorphisms in type 1 diabetes, Addison's disease and the polyglandular autoimmune syndrome type II. BMC Med Genet 2008;9:65.

3. Borgaonkar MR, Morgan DG. Primary biliary cirrhosis and type II autoimmune polyglandular syndrome. Can J Gastroenterol 1999;13:767-70.

4. Eisenbarth GS, Gottlieb PA. Autoimmune polyendocrine syndromes. N Engl J Med 2004;350:2068-79.

5. Förster G, Krummenauer F, Kühn I, Beyer J, Kahaly G. Polyglandular autoimmune syndrome type II: Epidemiology and forms of manifestation. Dtsch Med Wochenschr 1999;124:1476-81.

6. Betterle C, Lazzarotto F, Presotto F. Autoimmune polyglandular syndrome Type 2: The tip of an iceberg? Clin Exp Immunol 2004;137:225-33.

How to cite this article: Banzal S, Singhai A. Shock: A possible presenting manifestation of autoimmune polyendocrine syndrome type II. Indian J Crit Care Med 2014;18:326-7.

Source of Support: Nil, Conflict of Interest: None declared. 